\title{
METASÍNTESIS: DISCUSIÓN DE UN ABORDAJE METODOLÓGICO
}

\section{METASYNTHESIS: A DISCUSSION OF A METHODOLOGICAL APPROACH ${ }^{1}$}

\author{
Sonia Patricia Carreño Moreno* \\ Lorena Chaparro Díaz
}

\begin{abstract}
RESUMEN
El concepto de metasíntesis ha sido abordado por varios autores, coincidiendo en que es una forma de integrar hallazgos de investigación cualitativa con el fin de lograr una nueva interpretación que explica el fenómeno con un nivel mayor de evidencia científica. El presente artículo presenta las aproximaciones conceptuales y metodológicas de la propuesta de Sandelowski y Barroso, tomando como ejemplo la aplicación del método en un estudio de metasíntesis de calidad de vida en cuidadores de personas con enfermedad crónica.
\end{abstract}

Palabras clave: Metasíntesis, metodología, enfermería basada en la evidencia, enfermería.

\begin{abstract}
The concept of metasynthesis has been addressed by several authors who agree that is a way of integrating qualitative research findings in order to achieve a new interpretation that explains any given phenomenon with a higher level of scientific evidence. This paper presents the conceptual and methodological approach of the proposal by Sandelowski and Barroso, using as an example the application of this method in a study of meta-synthesis of quality of life among caregivers for people with chronic disease.
\end{abstract}

Key words: Metasynthesis, methodology, evidence based nursing, nursing.

Fecha recepción: 27/04/14 Fecha aceptación: 05/08/15

\footnotetext{
${ }^{1}$ Investigación financiada por la División de Investigación Universidad Nacional de Colombia sede Bogotá, programa apoyo a tesis de postgrado, código del proyecto 14554.

* Enfermera, Especialista en Prevención del Maltrato Infantil, Magíster en Enfermería con Énfasis en Cuidado al Paciente Crónico. Candidata a Doctora en Enfermería de la Universidad Nacional de Colombia. Profesora Auxiliar Facultad de Enfermería Universidad Nacional de Colombia. Email: spcarrenom@unal.edu.co

${ }^{* *}$ Enfermera, Doctora en Enfermería. Profesora Asistente Facultad de Enfermería Universidad Nacional de Colombia. Email: olchaparrod@unal.edu.co
} 


\section{INTRODUCCIÓN}

El término metasíntesis ha sido acuñado por varios autores con el fin de denominar formas de integrar hallazgos de investigación cualitativa (1-7). La metasíntesis se constituye en un método enmarcado en la práctica basada en la evidencia (PBE) o más específicamente denominada enfermería basada en la evidencia (EBE) (8-10), un movimiento científico que busca poner en marcha alternativas para la integración del conocimiento científico y hacerlo útil para la práctica $(11,12)$.

La metasíntesis cualitativa puede definirse como las teorías, narrativas globales, generalizaciones o traducciones interpretativas producidas para integrar o comparar los hallazgos obtenidos a partir de estudios cualitativos $(13,14)$; es una forma de revisión sistemática o integración de hallazgos de investigación cualitativa que son síntesis interpretativas de datos (15). El objetivo de la metasíntesis es crear grandes representaciones interpretativas de los hallazgos de estudios primarios, permaneciendo fiel a las interpretaciones de cada estudio particular (13). Independientemente de la denominación que le han dispuesto varios estudiosos, este género de investigación se refiere a un "estudio de estudios" que intenta lograr una nueva interpretación que va más allá de la suma de los resultados de cada estudio primario (12).

Aplicar una propuesta metodológica al estudio de un fenómeno es un desafío, pues concretar cada una de las acciones y buscar que respondan a la concepción filosófica, epistemológica y metodológica con que se concibió el abordaje seleccionado, requiere una continua reflexión, comparación y discusión que encause la síntesis de datos empíricos hacia la construcción de estructuras conceptuales con mayor nivel de evidencia científica. La preocupación que motivó este artículo es comparable con la de Ludvigsen et al. (16), quienes se preguntaron sobre los desafíos metodológicos de la síntesis de in- vestigación cualitativa en dos estudios de transición hospitalaria y desarrollaron un discusión acerca de cómo identificar la literatura, cómo realizar las búsquedas, cómo interpretar en tercer orden las experiencias de transición hospitalaria y si el estudio era realmente una metasíntesis.

El presente artículo presenta las aproximaciones conceptuales y metodológicas de la propuesta de Sandelowski y Barroso (17) tomando como ejemplo la aplicación del método en un estudio de metasíntesis de calidad de vida en cuidadores de personas con enfermedad crónica (18); ya que el discutir el abordaje metodológico que sobre metasíntesis proponen las autoras mencionadas es un aporte al conocimiento en términos de la aplicabilidad de la metodología y permite contar con conocimiento concreto acerca de los pasos a seguir en una metasíntesis.

\section{REQUERIMIENTOS PREVIOS A LA REALIZACIÓN DE UNA METASÍNTESIS}

1. Definición del fenómeno de interés. Es el primer elemento para centrar la atención de la búsqueda de literatura específica. Un fenómeno de enfermería es un aspecto de la realidad que puede ser percibido conscientemente o experimentado por el profesional de enfermería, son aspectos que reflejan el dominio o el territorio de la disciplina y se expresa a través de una descripción o la etiqueta para mostrar una idea acerca de un evento, una situación, un proceso, un grupo de eventos, o de un grupo de situaciones (19). Por lo general los fenómenos de interés para enfermería están asociados al paradigma de cuidado, el cual, para la investigación primaria que se usará como ejemplo de aplicación del método en este artículo, fue el de la experiencia de salud humana que propone Newman (20). Este paradigma permite reconocer que en el cuidador su experiencia tiene impacto en su calidad de vida, él se re-crea a medida que vive el cuidado, lo transforma como ser humano. La definición del fenómeno de interés permite 
al investigador buscar e identificar el método de síntesis de hallazgos cualitativos adecuado al tipo de fenómeno, resaltando que el tener claridad sobre la naturaleza del fenómeno de estudio facilita la toma de decisiones frente a la elección del método.

\section{Búsqueda exhaustiva en la literatura. Se} realiza para explorar el fenómeno de interés, los abordajes investigativos con que ha sido estudiado y el nivel de generación de conocimiento acerca del mismo, con el fin de determinar si existe un vacío en el conocimiento y si éste es suficiente para que se amerite su integración en una metasíntesis (21). En este paso se encontró que no existían metasíntesis publicadas en el fenómeno de interés y que existía una amplia literatura indexada en el mismo, por lo que se determinó que era un momento apto en la generación de conocimiento para desarrollarla. Cabe aclarar que no existe un límite de estudios que determinen si es pertinente sintetizar la evidencia, pues es el investigador con el conocimiento que tiene del fenómeno de interés, su recorrido conceptual, teórico y metodológico, quien considera el momento idóneo en que los hallazgos de investigación cualitativa de un fenómeno deben ser sintetizados.

3. Selección del método. Para realizar la selección del método a utilizar, es necesario hacer una búsqueda exhaustiva en la literatura que permita conocer el desarrollo del concepto de metasíntesis y su abordaje metodológico desde los diferentes autores. Para la elaboración de la metasíntesis mencionada se hizo revisión de diferentes métodos, como fue el propuesto por Noblit y Dwight denominado metaetnografía (3), que propone la combinación de estudios primarios etnográficos sobre un mismo tema, enfatizando en el uso de metáforas y relatos; el meta-estudio propuesto por Paterson et al. (4), que busca interpretar los resultados de investigaciones primarias, abriendo paso a la construcción de una teoría y el abordaje propuesto por
Sandelowski y Barroso (17), denominado metasíntesis cualitativa, que se refiere a un proceso de integración o comparación de hallazgos en la que se da especial importancia a las interpretaciones realizadas por los investigadores primarios, al igual que a las producciones científicas que reporten hallazgos en el fenómeno de interés.

Para la metasíntesis que se usó para ejemplificar el método discutido en este artículo, se seleccionó el método propuesto por Sandelowski y Barroso (17), pues ofrece elementos metodológicos contemplados en la metaetnografía como es la comparación constante y por la relevancia que se da a la interpretación de los investigadores primarios, permitiendo así que se pueda generar la reinterpretación que se constituye en la estructura conceptual que explica el fenómeno a estudiar.

La metaetnografía no fue seleccionada en razón a su fuerte influencia de la tradición investigativa etnográfica, pues aunque el autor expresa que es útil para sintetizar hallazgos cualitativos de diferentes tipos de estudios, el método considera la comparación constante de metáforas y relatos, los cuales son elementos propios de los estudios etnográficos, que no es usual encontrar en otras tradiciones cualitativas.

Por otra parte, fue descartado el metaestudio ya que su mayor utilidad se encuentra en la generación de teorías de rango medio, lo cual no fue un objetivo trazado para la metasíntesis que se desarrolló.

\section{CONSIDERACIONES METODOLÓGICAS EN LA METASÍNTESIS}

Con el método ya seleccionado, se contemplaron las siguientes consideraciones metodológicas:

\section{Selección de la pregunta de investigación.} En la revisión de literatura se pudo identificar el nivel de producción de conocimiento y las formas en que se había abordado el fenómeno de interés, lo que permitió 
formular la pregunta de investigación. Las metasíntesis se constituyen en estudios de enfermería basada en la evidencia, por ende fue útil pensar en una estructura de pregunta con los elementos del formato PICOT, el cual es un acróstico conformado por siglas en inglés que significa: P- Población o sujeto de interés, I- Intervención, C- Comparación, O- Resultados y T- Tiempo del estudio (22, 23). En la metasíntesis que ejemplifica la discusión en este artículo el formato PICOT fue (18): P- Cuidador Familiar de personas con enfermedad crónica, I- Significado de calidad de vida, C- No hay comparador, OReconstrucción del significado en el cuidado, T- Estudios de 1997 a 2012.

2. Selección de la muestra. El muestreo en metasíntesis está sujeto en primer lugar a la pregunta propuesta y en segundo lugar al conocimiento existente. Se recomienda que no se generen preguntas de investigación muy específicas, al igual que criterios de inclusión y exclusión muy restrictivos, pues se puede excluir información clave acerca del fenómeno (24). De esta manera, Sandelowski y Barroso sugieren algunos criterios de selección de la muestra como son: 1. Que las investigaciones primarias muestren que fueron abordadas con métodos cualitativos. 2. Que los hallazgos reportados se soporten en las fuentes primarias y que hayan pasado por un proceso de síntesis o análisis, es decir que no se presenten como datos aislados. 3. Que los reportes de investigación den cuenta de las consideraciones éticas para la investigación con seres humanos aplicadas a los mismos (25-27). Por otra parte, frente al tamaño de la muestra, no existe un estándar de estudios a seleccionar, puesto que es más relevante el contenido de la información que los estudios reportan que el número en sí mismo. Sin embargo, recomiendan un mínimo de 10 a 12 estudios (28). En la metasíntesis que se usa como ejemplo en este artículo (18), se definieron criterios de inclusión como: artículos acerca del fenómeno de interés de los últimos 15 años publicados en revistas indexadas, tesis de maestría o doctorado que aborden el fenómeno de interés disponibles en Biblioteca de la Universidad Nacional de Colombia; los criterios de exclusión fueron: publicaciones que no dieran cuenta de los criterios éticos en la investigación con seres humanos, publicaciones que presenten datos aislados sin interpretación o análisis y artículos de revisión.

Para la selección de la muestra se tuvieron en cuenta criterios para la búsqueda sistemática de literatura científica propuestos por Sandelowski y Barroso (17), considerando el fenómeno de interés y las características de los estudios primarios: el año de publicación, el sitio donde fue publicado y el diseño.

En el caso de la metasíntesis de calidad de vida de los cuidadores de personas con enfermedad crónica, los investigadores siguieron un itinerario de búsqueda sistemática de la literatura que cumplió con los siguientes pasos: 1. Definición de los descriptores o taxones de búsqueda, en donde se hizo un listado de términos clave y se cotejó con los árboles de taxones de los tesauros MeSH (29) y DesC (30), buscando los términos apropiados para realizar la búsqueda, así como las combinaciones y construcción de ecuaciones de búsqueda con el uso de operadores boleanos AND, OR, NOT. 2. Búsqueda en las bases de datos: CINHAL, Science Direct, DOAJ: Directory of open access journal, EBMR: Evidence Based Medicine Review, Global Health, Ebsco host, Springer, Biblioteca Virginia Henderson, Wiley Online Library, Dynamed, medline, LILACS y CUIDEN. 3. Preselección de los artículos por tiempo de publicación, diseño del estudio, título y abstract. 4. Traducción oficial de los artículos publicados en idiomas diferentes a la lengua materna de las autoras ${ }^{2}$. La Figura 1 ilustra el proceso de selección de muestra que siguió el estudio mencionado.

${ }^{2}$ La traducción oficial se refiere a un proceso en que personas certificadas por el estado traducen los textos, lo que garantiza que su contenido se mantenga fiel a lo que escribió el autor. 
Figura 1. Proceso de selección de la muestra.

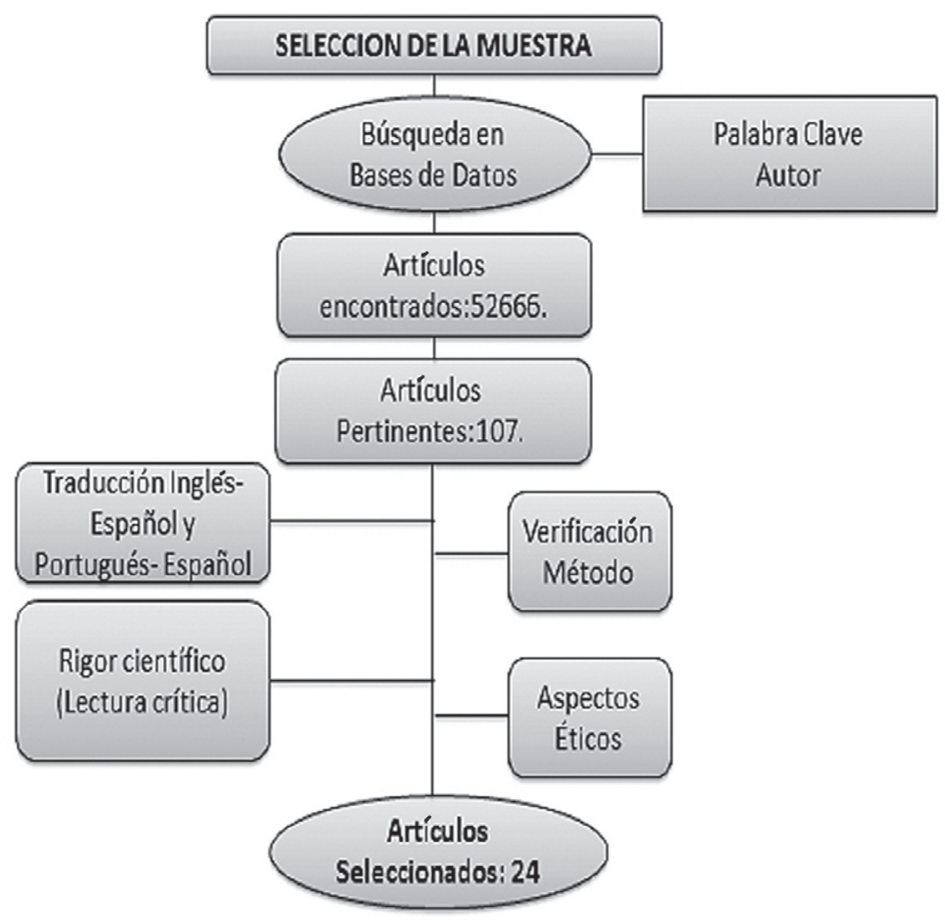

Fuente: Carreño Sonia. Metasíntesis de calidad de vida de cuidadores familiares de personas en situación de enfermedad crónica (31).

3. Revisión de calidad de los estudios seleccionados. Sandelowski y Barroso enfatizan en la calidad del hallazgo sobre la "calidad" del estudio. Afirman que aunque se presenten errores metodológicos en el estudio por discrepancias entre lo planteado en su diseño y el método realmente usado, los hallazgos no se invalidan. Es de aclarar que esto no significa que los estudios a incluir en la metasíntesis carezcan de rigor científico, pues las autoras definieron un sistema de tipología de hallazgos para revisar la información de interés (17). De esta manera se encuentran los no hallazgos (datos aislados presentados literalmente sin interpretación), listas o inventarios temáticos (datos con mínima interpretación) y hallazgos sintetizados (transformaciones interpretativas de datos originales). Por otra parte, las autoras declaran la importancia de hacer lectura crítica de los estudios antes de ser seleccionados y proponen algunos parámetros para hacerla, los cuales se basan en la crítica del problema, el objetivo, la revisión de literatura, el método, el muestreo, técnicas de recolección y gestión de datos, hallazgos, discusión, validez, ética y formato de los informes de investigación cualitativa con unos parámetros de apreciación determinados que pretenden evaluar la calidad del estudio $(17,32,33)$.

En la metasíntesis de calidad de vida de cuidadores de personas con enfermedad crónica (18), la calidad de los hallazgos fue verificada por las dos autoras en un proceso continuo de comparación y crítica que concluyó en la selección de 24 estudios primarios. Además, la discusión sobre la calidad de los hallazgos se extendió a un grupo re- 
conocido de investigación conformado por cinco integrantes con amplia trayectoria en el estudio de fenómenos relacionados con la calidad de vida y con los cuidadores de personas con enfermedad crónica, buscando interpretaciones válidas de los hallazgos como un elemento fundamental en la metasíntesis cualitativa (34).

\section{Técnica para el proceso de metasíntesis} cualitativa. Esta técnica transforma los hallazgos en conceptos (7). Dentro de la técnica se incluyen herramientas de análisis y síntesis (Figura 2):

a. Creación de una taxonomía de los hallazgos: Debe desarrollarse inductivamente a partir de códigos y categorías que permiten agrupar los hallazgos, mostrando sus propiedades teóricas y conceptuales. La aplicación de este paso metodológico en la metasíntesis mencionada, la taxonomía, emergió a partir de códigos descriptores, códigos nominales, categorías, variables y variable central que se desarrollaron con la ayuda de la herramienta informática Atlas Ti versión 6.0. El proceso de creación de la taxonomía se desarrolló en los siguientes paso: 1. Una autora realizó trabajo en los códigos descriptores y nominales. 2. Se desarrolló una discusión entre las dos autoras acerca de la codificación inicial que incluyó la recodificación de algunos de los hallazgos. 3. Se hizo comparación constante con la ayuda de memos, buscando reducir los códigos nominales a categorías, luego las categorías a variables $\mathrm{y}$ al final a una variable que agrupara los hallazgos. b. Uso de comparaciones continuas: Se realizan con el fin de clarificar los atributos que definen el fenómeno y cómo se interrelaciona con otros fenómenos afines. Los resultados de esta fase se pueden presentar a través de diagramas que muestren una descripción conceptual amplia o un modelo.

En la metasíntesis mencionada, la comparación constante se dio a partir de la formulación de memos; además se realizaron diferentes tipos de diagramas durante todo el proceso de comparación que dieron lugar a un diagrama total que muestra el fenómeno, los factores condicionantes y la relación con otros fenómenos; tanto los memos como los diagramas se desarrollaron con la herramienta Atlas Ti antes mencionada (31).

c. Traducción de conceptos in vivo: Se utiliza para aquellos hallazgos que constituyen un concepto central y los cuales fueron elaborados a partir de los datos obtenidos de las fuentes primarias (participantes). En la metasíntesis mencionada emergieron conceptos in vivo dada su importancia y carácter central como fueron sentimientos ambiguos y sobrecarga (31).

d. Uso de conceptos importados: Se refiere al uso de conceptos previos a cada investigación primaria. En la metasíntesis mencionada no se hizo uso de conceptos importados. 
Figura 2. Proceso de elaboración de una metasíntesis cualitativa.

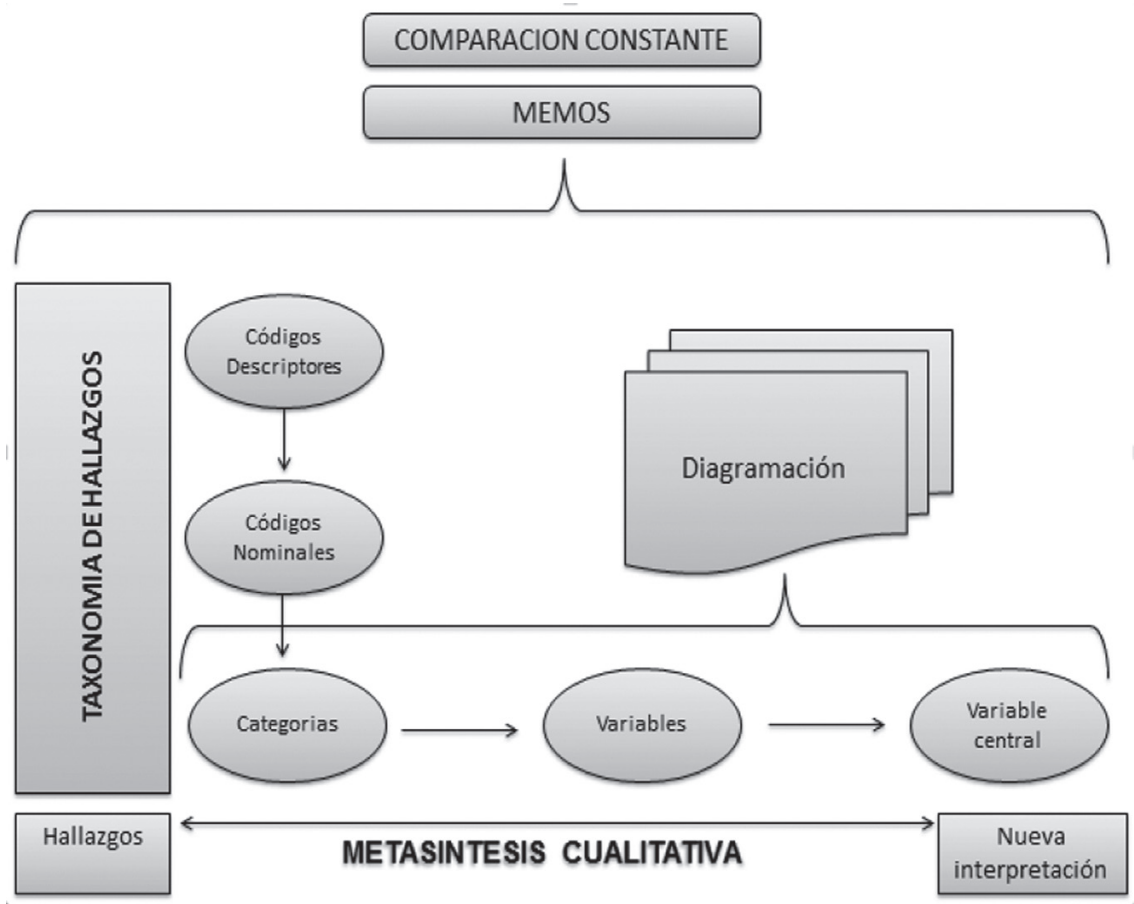

Fuente: Carreño Sonia. Metasíntesis de calidad de vida de cuidadores familiares de personas en situación de enfermedad crónica (31).

\section{CONSIDERACIONES FINALES}

La metasíntesis cualitativa es una poderosa herramienta en la enfermería basada en la evidencia, que permite construir nuevo conocimiento para mejorar la práctica de enfermería en ambientes clínicos y comunitarios, con enfoques basados en la experiencia del cuidado de la salud humana, los cuales se posicionan en las visiones del mundo de la interacción y de la simultaneidad, tan necesarias en un ambiente de la salud carente de sensibilidad humana.

En el acervo de conocimiento de enfermería generado a través de la investigación cualitativa es bastante nutrido, lo que debe motivar a los investigadores del siglo XXI a generar novedad en el conocimiento con la reinterpretación teórica y su entendimiento para mejorar las condiciones del cuidado de la salud de las personas, proponer estrategias para incorporarlas en los lineamientos de la política institucional del cuidado de la salud y transferir ese conocimiento nuevo a las nuevas generaciones de enfermeros convencidos de una práctica sustentada en la investigación y no solo en la práctica tradicional.

La producción en los últimos años de investigaciones con abordaje cualitativo hace de la metasíntesis un método efectivo de transformación de hallazgos en estructuras conceptuales y teóricas más complejas que permiten una mejor explicación y compren- 
sión de los fenómenos de interés para la disciplina.

El proceso de metasíntesis cualitativa propuesto por Sandelowski y Barroso (17) da cuenta de años de experiencia investigativa en estas técnicas, de consensos de expertos y de evidencia científica que lo soporta, por lo que se constituye en una herramienta idónea para el abordaje de este tipo de estudios.

Los diferentes abordajes metodológicos de la metasíntesis son lineamientos útiles para la nueva era de la investigación, en la que el reto es la creatividad puesta a prueba cuando se refleja fielmente los hallazgos de muchos investigadores previos, se consolida en nueva estructura de conocimiento bajo un paradigma integral y unitario y sobre todo cuando se construye un microproceso para reunir todos los elementos del rompecabezas de la metasíntesis con el rigor que tiene la investigación cualitativa y que ha demostrado ser válida en el conocimiento de la ciencia.

\section{REFERENCIAS}

1. Estabrooks CA, Field PA, Morse JM. Aggregating Qualitative Findings: An Approach to Theory Development. Qual Health Res. 1994 ;4(4): 503-11.

2. Jensen LA, Allen MN. Meta-Synthesis of Qualitative Findings. Qual Health Res. 1996; 6(4): 553-60.

3. Noblit G, Dwight H. Meta-Ethnography: Synthesizing Qualitative Studies. Qualitative Research Methods. 11th ed. London: Sage Publications; 1988. 108 p.

4. Paterson BL, Thorne SE, Canam C, Jillings C. Meta-Study of Qualitative Health Research: A Practical Guide to Meta-Analysis and Meta-Synthesis. Thousand Oaks, CA: SAGE Publications; 2001. 162 p.

5. Roux G, Dingley CE, Bush HA. Inner strength in women: metasynthesis of qualitative findings in theory development. J Theory Constr Test. 2002; 6(1): 86-93.

6. Beck CT. Mothering multiples: a meta-synthesis of qualitative research. MCN Am J Matern Child Nurs. 2002; 27(4): 214-21.

7. Margarete S, Docherty S, Emden C. Focus on Qualitative Methods Qualitative Metasynthesis: Issues and Techniques. Res Nurs Health. 1997; 20: 365-71.

8. Gálvez A. Enfermería basada en la evidencia: cómo incorporar la investigación a la práctica de los cuidados. 2a ed. Granada: Fundación Index; 2007. 364 p.

9. Gálvez A. Actualidad de la Enfermería Basada en la Evidencia. Superando la "Evidencia Científica": la Metasíntesis. Index de Enfermería. 2003; 40-41: 7-8.

10. Gálvez A. Evidencias, pruebas científicas y enfermería. Reflexión en voz baja y pensamientos inconfesables. Enfermería Glob. 2003; 2(2): 1-13.

11. Mahtani V, Axpe M, Serrano P, González I, Fernández E. Metodología para incorporar los estudios cualitativos en la evaluación de tecnologías sanitarias. [Internet]. Gobierno de Canarias. 2006 [citado 13 ago 2015]. Disponible en: http://www3. gobiernodecanarias.org/sanidad/scs/ contenidoGenerico.jsp?idDocumen$\mathrm{t}=\mathrm{c} 71 \mathrm{cdabd}-1 \mathrm{fc} 5-11 \mathrm{e} 0-964 \mathrm{e}-\mathrm{f5f3323ccc-}$ 4d\&idCarpeta $=993 a 9 b 1 d-7 a e d-11 e 4-a 62$ a-758e414b4260

12. Thorne S, Jensen L, Kearney MH, Noblit G, Sandelowski M. Qualitative metasynthesis: reflections on methodological orientation and ideological agenda. Qual Health Res. 2004; 14(10): 1342-65.

13. Xu Y. Methodological issues and challenges in data collection and analysis of qualitative meta-synthesis. Asian Nurs Res. 2008; 2(3): 173-83.

14. Barroso J, Gollop CJ, Sandelowski M, Meynell J, Pearce PF, Collins LJ. The challenges of searching for and retrieving 
qualitative studies. West J Nurs Res. 2003; 25(2): 153-78.

15. Sandelowski M, Barroso J. Toward a metasynthesis of qualitative findings on motherhood in HIV-positive women. Res Nurs Health. 2003; 26(2): 153-70.

16. Ludvigsen MS, Hall EOC, Meyer G, Fegran L, Aagaard H, Uhrenfeldt L. Using Sandelowski and Barroso's Metasynthesis Method in Advancing Qualitative Evidence. Qual Health Res. En prensa 2015.

17. Sandelowski M, Barroso J. Handbook for Synthesizing Qualitative Research. New York: Springer Publishing Company; 2007. 284 p.

18. Carreño Moreno SP, Chaparro Díaz L. Reconstruyendo el significado de calidad de vida de los cuidadores en el cuidado: una metasíntesis. Av en Enfermería [Internet]. Universidad Nacional de Colombia; 2015 Aug 5 [cited 13 ago 2015]; 33(1): 55-66. Disponible en: http://www. scielo.org.co/scielo.php?script=sci_arttext\&pid=S0121-45002015000100007\&l$\mathrm{ng}=\mathrm{en} \& \mathrm{nrm}=\mathrm{iso} \& \operatorname{lng}=\mathrm{es}$

19. Meleis AI. Theoretical Nursing: Development and Progress. 5th ed. Philadelphia: Wolters Kluwer Health/ Lippincott Williams \& Wilkins; 2011.672 p.

20. Newman MA, Smith MC, Pharris MD, Jones $\mathrm{D}$. The focus of the discipline revisited. Adv Nurs Sci. 2008; 31(1): E16-27.

21. Sandelowski M, Barroso J. Finding the findings in qualitative studies. J Nurs Scholarsh. 2002; 34(3): 213-9.

22. Mamédi C, Mattos CA de, Moacyr C. The PICO strategy for the research question construction and evidence search. Rev Lat Am Enfermagem. 2007; 15(3): 50811.

23. Stone PW. Popping the (PICO) question in research and evidence-based practice. Appl Nurs Res. 2002; 15(3): 197-8.

24. Bondas T, Hall EOC. A decade of metasynthesis research in health sciences: A meta-method study. Int J Qual Stud
Health Well-being. 2007; 2(2): 101-13.

25. Sandelowski M, Barroso J. Creating metasummaries of qualitative findings. Nurs Res. 2003; 52(4): 226-33.

26. Sandelowski M, Barroso J, Voils CI. Using qualitative metasummary to synthesize qualitative and quantitative descriptive findings. Res Nurs Health. 2007; 30(1): 99-111.

27. Sandelowski M. "Meta-Jeopardy": the crisis of representation in qualitative metasynthesis. Nurs Outlook. 2006; 54(1): 106.

28. DiGirolamo AM, Salgado de Snyder N. Women as primary caregivers in Mexico: challenges to well-being. Salud Publica Mex. 2008; 50(6): 516-22.

29. NCBI. Home - MeSH - NCBI [Internet]. Bethesda, MD: NCBI; [citado 13 ago 2015]. Disponible en: http://www.ncbi. nlm.nih.gov/mesh

30. Biblioteca Virtual en Salud. DeCS - Descriptores en Ciencias de la Salud [Internet]. São Paulo: BIREME; [citado 13 ago 2015]. Disponible en: http://decs.bvs. br/E/homepagee.htm

31. Carreño S. Metasíntesis de calidad de vida en Cuidadores Familiares de Personas en Situación de Enfermedad Crónica [Tesis de Maestría en Internet]. Bogotá: Universidad Nacional de Colombia; 2012 [citado 13 jul 2015]. 157 p. Disponible en: http://www.bdigital.unal.edu.co/8564/

32. Sandelowski M, Barroso J. Classifying the findings in qualitative studies. Qual Health Res. 2003; 13(7): 905-23.

33. Sandelowski M, Barroso J. Reading Qualitative Studies. Int J Qual Methods [Internet]. 2002 [citado 13 ago 2015];1(1). Disponible en: https://www.ualberta. ca/ iiqm/backissues/1_1Final/pdf/sandeleng.pdf

34. Sandelowski M. When a cigar is not just a cigar: alternative takes on data and data analysis. Res Nurs Health. 2011; 34(4): 342-52. 